\title{
Information Theory of Cognitive Radio System
}

\author{
F. G. Awan ${ }^{1}$, N. M. Sheikh ${ }^{1}$ and M. F. Hanif * \\ ${ }^{1}$ University of Engineering and Technology \\ Lahore, 54890 Pakistan \\ *University of the Punjab \\ Quaid-e-Azam Campus \\ 54590, Lahore \\ Pakistan
}

\section{Introduction}

Cognitive radio $(\mathrm{CR})$ carries bright prospects for very efficient utilization of spectrum in future. Since cognitive radio is still in its early days, many of its theoretical limits are yet to be explored. In particular, determination of its maximum information rates for the most general case is still an open problem. Till today, many cognitive channel models have been presented. Either achievable or maximum bit rates have been evaluated for each of these. This chapter will summarize all the existing results, makes a comparison between different channel models and draw useful conclusions.

The scarcity of the radio frequency (RF) spectrum along with its severe under utilization, as suggested by various government bodies like the Federal Communications Commission (FCC) in USA and Ofcom in UK like Rafsh [1] and [2] has triggered immense research activity on the concept of $\mathrm{CR}$ all over the world. Of the many facets that need to be dealt with, the information theoretic modeling of CR is of core importance, as it helps predict the fundamental limit of its maximum reliable data transmission. The information theoretic model proposed in [3] represents the real world scenario that the CR will have to encounter in the presence of primary user (PU) devices. Authors in [3] characterize the CR system as an interference channel with degraded message sets (IC-DMS), since the spectrum sensing nature of the CR may enable its transmitter (TX) to know PU's message provided the PU is in close proximity of the CR. Elegantly using a combination of rate-splitting [4] and Gel'fand Pinsker (GP) [5] coding, [3] has given an achievable rate region of the so called CR-channel or IC-DMS. Further, in [3] time sharing is performed between the two extreme cases when either the CR dedicates zero power ("highly polite") or full power ("highly rude") to its message. A complete review of information theoretic studies can be found in [6] and [7].

This chapter then discusses outer-bounds to the individual rates and the conditions under which these bounds become tight for the symmetric Gaussian CR channel in the low interference gain regime. The CR transmitter is assumed to use dirty paper coding while deriving the outer-bounds. The capacity of the CR channel in the low interference scenario is 
known when the CR employs "polite" approach by devoting some portion of its power to transmit PU's message that will help calculating quality of service for the CR users. Then, we will focus on the scenario when the CR goes for the "rude" approach i.e., does not relay PU's message and tries to maximize its own rates only. It will be derived that when both CR and the PU operate in low interference gain regime, then treating interference as additive noise at the PU receiver and doing dirty paper coding at the CR is nearly optimal.

\section{Cognitive Radio Network Paradigms}

Since its introduction in [8], the definition of cognitive radio has evolved over the years. Consequently, different interpretations of cognitive radio and different visions for its future exist today. In this section we describe a few communication models that have been proposed for cognitive radio. We broadly classify them into overlay or known interference models, underlay or interference avoidance models.

\section{Underlay Paradigm:}

The underlay paradigm encompasses techniques that allow communication by the cognitive radio assuming it has knowledge of the interference caused by its transmitter to the receivers of all noncognitive users [7]. In this setting the cognitive radio is often called a secondary user which cannot significantly interfere with the communication of existing (typically licensed) users, who are referred to as primary users. Specifically, the underlay paradigm mandates that concurrent noncognitive and cognitive transmissions may occur only if the interference generated by the cognitive devices at the noncognitive receivers is below some acceptable threshold. The interference constraint for the noncognitive users may be met by using multiple antennas to guide the cognitive signals away from the noncognitive receivers, or by using a wide bandwidth over which the cognitive signal can be spread below the noise floor, then despread at the cognitive receiver. The latter technique is the basis of both spread spectrum and ultrawideband (UWB) communications.

The interference caused by a cognitive transmitter to a noncognitive receiver can be approximated via reciprocity if the cognitive transmitter can overhear a transmission from the cognitive receiver's location. Alternatively, the cognitive transmitter can be very conservative in its output power to ensure that its signal remains below the prescribed interference threshold. In this case, since the interference constraints in underlay systems are typically quite restrictive, this limits the cognitive users to short range communications.

While the underlay paradigm is most common in the licensed spectrum (e.g. UWB underlays many licensed spectral bands), it can also be used in unlicensed bands to provide different classes of service to different users.

\section{Overlay Paradigm:}

The enabling premise for overlay systems is that the cognitive transmitter has knowledge of the noncognitive users' codebooks and its messages as well [7]. The codebook information could be obtained, for example, if the noncognitive users follow a uniform standard for communication based on a publicized codebook. Alternatively, they could broadcast their codebooks periodically. A noncognitive user message might be obtained by decoding the message at the cognitive receiver. However, the overlay model assumes the noncognitive message is known at the cognitive transmitter when the noncognitive user begins its 
transmission. While this is impractical for an initial transmission, the assumption holds for a message retransmission where the cognitive user hears the first transmission and decodes it, while the intended receiver cannot decode the initial transmission due to fading or interference. Alternatively, the noncognitive user may send its message to the cognitive user (assumed to be close by) prior to its transmission. Knowledge of a noncognitive user's message and/or codebook can be exploited in a variety of ways to either cancel or mitigate the interference seen at the cognitive and noncognitive receivers. On the one hand, this information can be used to completely cancel the interference due to the noncognitive signals at the cognitive receiver by sophisticated techniques like dirty paper coding [9]. On the other hand, the cognitive users can utilize this knowledge and assign part of their power for their own communication and the remainder of the power to assist (relay) the noncognitive transmissions. By careful choice of the power split, the increase in the noncognitive user's signal-to-noise power ratio (SNR) due to the assistance from cognitive relaying can be exactly offset by the decrease in the noncognitive user's SNR due to the interference caused by the remainder of the cognitive user's transmit power used for its own communication. This guarantees that the noncognitive user's rate remains unchanged while the cognitive user allocates part of its power for its own transmissions. Note that the overlay paradigm can be applied to either licensed or unlicensed band communications. In licensed bands, cognitive users would be allowed to share the band with the licensed users since they would not interfere with, and might even improve, their communications. In unlicensed bands cognitive users would enable a higher spectral efficiency by exploiting message and codebook knowledge to reduce interference.

\section{Interweave Paradigm:}

The 'interweave' paradigm is based on the idea of opportunistic communication, and was the original motivation for cognitive radio [10]. The idea came about after studies conducted by the FCC [8] and industry [2] showed that a major part of the spectrum is not utilized most of the time. In other words, there exist temporary space-time frequency voids, referred to as spectrum holes, that are not in constant use in both the licensed and unlicensed bands.

These gaps change with time and geographic location, and can be exploited by cognitive users for their communication. Thus, the utilization of spectrum is improved by opportunistic frequency reuse over the spectrum holes. The interweave technique requires knowledge of the activity information of the noncognitive (licensed or unlicensed) users in the spectrum. One could also consider that all the users in a given band are cognitive, but existing users become primary users, and new users become secondary users that cannot interfere with communications already taking place between existing users.

To summarize, an interweave cognitive radio is an intelligent wireless communication system that periodically monitors the radio spectrum, intelligently detects occupancy in the different parts of the spectrum and then opportunistically communicates over spectrum holes with minimal interference to the active users. For a fascinating motivation and discussion of the signal processing challenges faced in interweave cognitive radio, we refer the reader to [11].

Table 1 [12] summarizes the differences between the underlay, overlay and interweave cognitive radio approaches. While underlay and overlay techniques permit concurrent cognitive and noncognitive communication, avoiding simultaneous transmissions with 
noncognitive or existing users is the main goal in the interweave technique. We also point out that the cognitive radio approaches require different amounts of side information: underlay systems require knowledge of the interference caused by the cognitive transmitter to the noncognitive receiver(s), interweave systems require considerable side information about the noncognitive or existing user activity (which can be obtained from robust primary user sensing) and overlay systems require a large amount of side information (non-causal knowledge of the noncognitive user's codebook and possibly its message). Apart from device level power limits, the cognitive user's transmit power in the underlay and interweave approaches is decided by the interference constraint and range of sensing, respectively. While underlay, overlay and interweave are three distinct approaches to cognitive radio, hybrid schemes can also be constructed that combine the advantages of different approaches. For example, the overlay and interweave approaches are combined in [7].

Before launching into capacity results for these three cognitive radio networks, we will first review capacity results for the interference channel. Since cognitive radio networks are based on the notion of minimal interference, the interference channel provides a fundamental building block to the capacity as well as encoding and decoding strategies for these networks.

\begin{tabular}{|c|c|c|}
\hline Underlay & Overlay & Interweave \\
\hline $\begin{array}{l}\text { Channel Side Information: Cognitive } \\
\text { (secondary) transmitter knows the channel } \\
\text { strengths to noncognitive (primary) } \\
\text { receiver(s). }\end{array}$ & $\begin{array}{l}\text { Codebook Side Information: Cognitive } \\
\text { nodes know channel gains, codebooks and } \\
\text { the messages of the noncognitive users }\end{array}$ & $\begin{array}{l}\text { Activity Side Information: Cognitive user } \\
\text { knows the spectral holes in space, time, or } \\
\text { frequency when the noncognitive user is not } \\
\text { using these holes. }\end{array}$ \\
\hline $\begin{array}{l}\text { Cognitive user can transmit simultaneously } \\
\text { with noncognitive user as long as } \\
\text { interference caused is below an acceptable } \\
\text { limit. }\end{array}$ & $\begin{array}{l}\text { Cognitive user can transmit simultaneously } \\
\text { with noncognitive user; the interference to } \\
\text { noncognitive user can be offset by using } \\
\text { part of the cognitive user's power to relay } \\
\text { the noncognitive user's message. }\end{array}$ & $\begin{array}{l}\text { Cognitive user transmits simultaneously } \\
\text { with a noncognitive user only in the event of } \\
\text { a false spectral hole detection. }\end{array}$ \\
\hline $\begin{array}{l}\text { Cognitive user's transmit power is limited by } \\
\text { the interference constraint. }\end{array}$ & $\begin{array}{l}\text { Cognitive user can transmit at any power, } \\
\text { the interference to noncognitive users can } \\
\text { be offset by relaying the noncognitive user's } \\
\text { message. }\end{array}$ & $\begin{array}{l}\text { Cognitive user's transmit power is limited by } \\
\text { the } \\
\text { range of its spectral hole sensing. }\end{array}$ \\
\hline
\end{tabular}

Table 1. Comparison of underlay, overlay and interweave cognitive radio techniques.

\section{Interference-Mitigating Cognitive Behavior: The Congnitive Radio Channel}

This discussion is has been taken from Natasha's paper. This consideration is simplest possible scenario in which a cognitive radio could be employed. Assume there exists a primary transmitter and receiver pair (S1 - R1), as well as the cognitive secondary transmitter and receiver pair (S2 - R2). As shown in Fig. 1.(a), there are three possibilities for transmitter cooperation in these two point-to-point channels. We have chosen to focus on transmitter cooperation because such cooperation is often more insightful and general than receiver-side cooperation $[12,13]$. Thus assume that each receiver decodes independently. Transmitter cooperation in this figure is denoted by a directed double line. These three channels are simple examples of the cognitive decomposition of wireless networks seen in [14]. The three possible types of transmitter cooperation in this simplified scenario are: 
1. Competitive behavior: The two transmitters transmit independent messages. There is no cooperation in sending the messages, and thus the two users compete for the channel. This is the same channel as the 2 sender, 2 receiver interference channel $[14,15]$.

2. Cognitive behavior: Asymmetric cooperation is possible between the transmitters. This asymmetric cooperation is a result of S2 knowing S1's message, but not vice-versa. As a first step, we idealize the concept of message knowledge: whenever the cognitive node S2 is able to hear and decode the message of the primary node S1, we assume it has full a priori knowledge. This is called the genie assumption, as these messages could have been given to the appropriate transmitters by a genie. The one way double arrow indicates that S2 knows S1's message but not vice versa.

This is the simplest form of asymmetric non-causal cooperation at the transmitters. Usage of the term cognitive behavior is to emphasize the need for S2 to be a "smart" device capable of altering its transmission strategy according to the message of the primary user. We can motivate considering asymmetric side information in practice in three ways:

- Depending on the device capabilities, as well as the geometry and channel gains between the various nodes, certain cognitive nodes may be able to hear and/or obtain the messages to be transmitted by other nodes. These messages would need to be obtained in real time, and could exploit the geometric gains between cooperating transmitters relative to receivers in, for example, a 2 phase protocol [3].

- In an Automatic Repeat reQuest (ARQ) system, a cognitive transmitter, under suitable channel conditions (if it has a better channel to the primary transmitting node than the primary receiver), could decode the primary user's transmitted message during an initial transmission attempt. In the event that the primary receiver was not able to correctly decode the message, and it must be retransmitted, the cognitive user would already have the to-betransmitted message, or asymmetric side information, at no extra cost (in terms of overhead in obtaining the message).

- The authors in [16] consider a network of wireless sensors in which a sensor S2 has a better sensing capability than another sensor S1 and thus is able to sense two events, while $\mathrm{S} 1$ is only able to sense one. Thus, when they wish to transmit, they must do so under an asymmetric side-information assumption: sensor S2 has two messages, and the other has just one.

3. Cooperative behavior: The two transmitters know each others' messages (two way double arrows) and can thus fully and symmetrically cooperate in their transmission. The channel pictured in Fig. 1. (c) may be thought of as a two antenna sender, two single antenna receivers broadcast channel [17].

Many of the classical, well known information theoretic channels fall into the categories of competitive and cooperative behavior. For more details, we refer the interested reader to the cognitive network decomposition theorem of [13] and [18]. We now turn to the much less studied behavior which spans and in a sense interpolates between the symmetric cooperative and competitive behaviors. We call this behavior asymmetric cognitive 
behavior. In this section we will consider one example of cognitive behavior: a two sender, two receiver (with two independent messages) interference channel with asymmetric and a priori message knowledge at one of the transmitters, as shown in Fig. 1. (b).

Certain asymmetric (in transmitter cooperation) channels have been considered in the literature: for example in [19], the capacity region of a multiple access channel with asymmetric cooperation between the two transmitters is computed. The authors in [20] consider a channel which could involve asymmetric transmitter cooperation, and explore the conditions under which the capacity of this channel coincides with the capacity of the channel in which both messages are decoded at both receivers. In [21, 18] the authors introduced the cognitive radio channel, which captures the most basic form of asymmetric transmitter cooperation for the interference channel. We now study the information theoretic limits of interference channels with asymmetric transmitter cooperation, or cognitive radio channels.

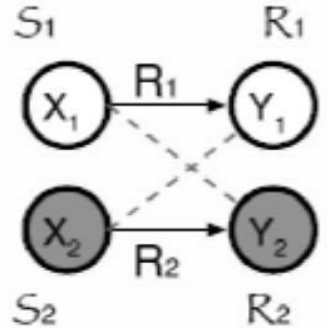

(a)

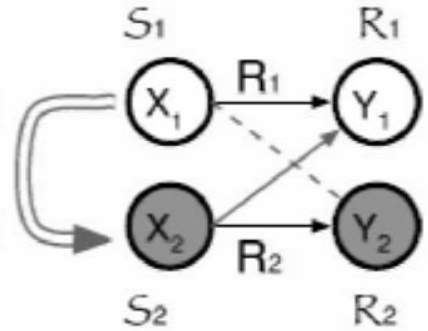

(b)

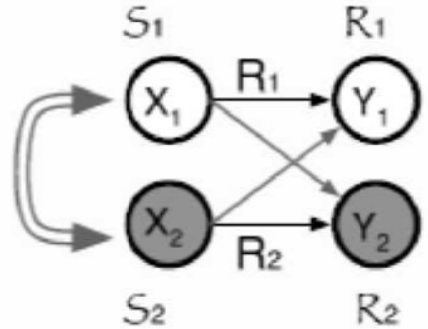

(c)

Fig. 1. (a) Competitive behavior, the interference channel. The transmitters may not cooperate. (b) Cognitive behavior, the cognitive radio channel. Asymmetric transmitter cooperation. (c) Cooperative behavior, the two antenna broadcast channel. The transmitters, but not the receivers, may fully and symmetrically cooperate.

The channel is thus expressed via following pair of equations:

$$
\begin{aligned}
& Y_{p}=X_{p}+a X_{s}+Z_{p} \\
& Y_{s}=b X_{p}+X_{s}+Z_{s}
\end{aligned}
$$

While deriving the channel capacity, an assumption of low interference- gain has been made. Low interference regime corresponds to the scenario where the cognitive user is assumed to be near its own base station rather than that of primary user, which normally is the case. When applied to the interference channel in standard form, this situation corresponds to $\mathrm{a} \leq 1$.

At the same time the two devices are assumed to work in an environment where the coexistence conditions exist, ensuring that cognitive radio generates no interference with the primary user in its vicinity and the primary receiver is a single user decoder. With all these assumptions, the channel is named as the cognitive $(1, \mathrm{a}, \mathrm{b}, 1)$ channel. The capacity $R s$ (in bps) of the cognitive radio under the conditions existing as above is expressed in a closed form relation as: 


$$
R_{S}^{*}=\frac{1}{2} \log \left(1+\left(1-\alpha^{*}\right) P_{S}\right) \quad \text { provided } a \leq 1
$$

where $\alpha^{*} \in[0,1]$ and its value is determined using the following arguments:

To ensure that the primary user remains unconscious of the presence of the cognitive device and communicates at a rate of $1 / 2 \log \left(1+P_{p}\right)$, the maximum achievable rate $R_{p}^{*}$ of the primary system is found to be:

$$
\frac{1}{2} \log \left(1+\frac{\left(\sqrt{P_{p}}+a \sqrt{\alpha P_{S}}\right)^{2}}{1+a^{2}(1-\alpha) P_{S}}\right)=\frac{1}{2} \log \left(1+P_{p}\right)=R_{p}^{*}
$$

Now for $0<a<1$, using Intermediate Value Theorem, this quadratic equation in $a$ always has a unique root in $[0,1]$ :

$$
\alpha^{*}=\left(\frac{\sqrt{P_{p}}\left(\sqrt{1+a^{2} P_{S}\left(1+P_{p}\right)}-1\right)}{a \sqrt{P_{S}}\left(1+P_{p}\right)}\right)^{\frac{1}{2}}
$$

It is to be noted that the above capacity expressions hold for any $b \in R$. For detailed proofs the reader is referred to [8].

A few important points are worth mentioning here. Since the cognitive radio knows both $m p$ (the message to be transmitted by primary user) and $m s$ (the message to be transmitted by the cognitive device), it generates its codeword $X_{n s}$ such that it also incorporates the codeword $X_{n p}$ to be generated by the primary user. By doing so, the cognitive device can implement the concept of dirty paper coding that helps it mitigating the interference caused by the primary user at the cognitive receiver. Thus the cognitive device performs superposition coding as follows:

$$
X_{S}^{n}=\widehat{X}_{S}^{n}+\sqrt{\frac{\alpha P_{s}}{P_{p}}} X_{p}^{n}
$$

Where $\widehat{X}_{S}^{n}$ encodes $m_{s}$ and is generated by performing dirty paper coding, treating $\left(b+\sqrt{\frac{\alpha P_{S}}{P_{p}}}\right) X_{p}^{n}$ as a known interference that will affect the secondary receiver. It is evident from (6) that the secondary device uses part of its power $\alpha$ Ps to relay the primary user's message to the primary receiver. This relaying of message from the secondary user results in an elevated value of SNR at the primary receiver. At the same time, the secondary user's message with power $(1-\alpha) P_{s}$, transmitted towards the primary receiver balances the increase in SNR and as a result the primary device remains completely oblivious to the presence of the cognitive user. This approach has been named as selfless approach in [23].

In [8], results corresponding to high interference regime $a>1$ have also been presented as ancillary conclusions. But such results are of not much significance as they present the 
scenario of high interference caused by the secondary user which is an event with low probability of occurrence.

Similar results have also been obtained in [3], [16] and [15]. The results in [15] correspond to the selfish approach of [23] and thus represent an upper bound on information rates of cognitive radio but with interference, because in this case the cognitive user does not spend any of its power in relaying the message to the primary receiver. Similarly authors in [16] have shown that, in the Gaussian noise case, their capacity region is explicitly equal to that of [8] and, numerically, to that of [23]. Very recently [24] has also extended the results of [8] to the case of Gaussian Multiple Access channel (MAC) with $n$ cognitive users. [24] has simply used the results for a general MAC channel i.e., the achievable rate for n-users is the sum of the achievable rates of individual users. Using this, together with the result of [8], it has determined the achievable rate region of a Gaussian MAC cognitive channel.

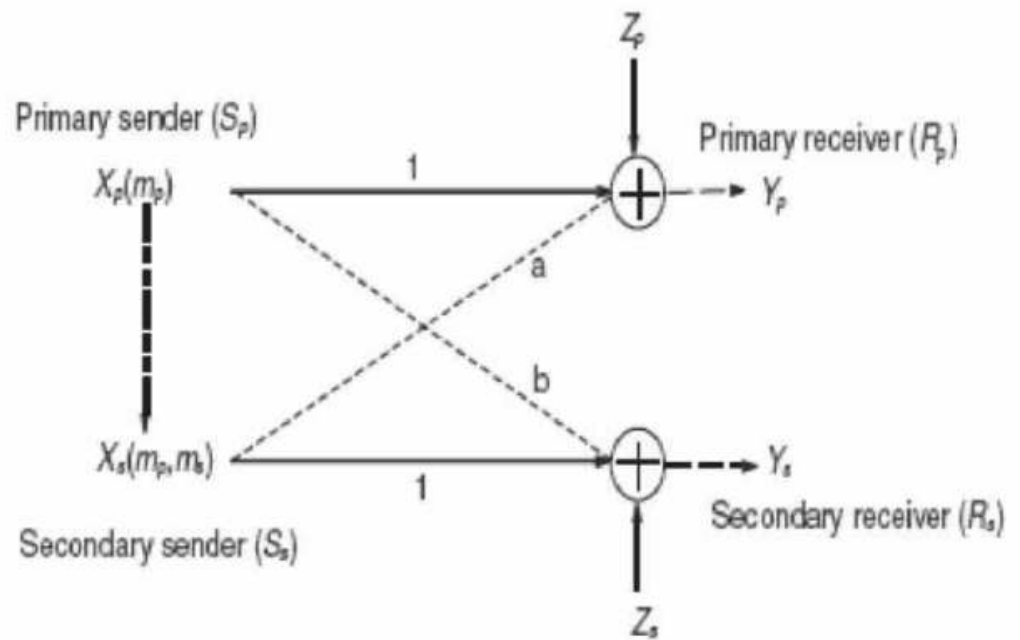

Fig. 2. The Gaussian interference channel in its standard form

\section{Interference Avoiding Channel}

This channel model, as devised in [22] and [6], works on the principle of opportunistic communication i.e., the secondary communication takes place only when the licensed user is found to be idle and a spectrum hole is detected. Thus this model conforms to the basic requirement of the cognitive device not interfering with the licensed users. The secondary sender $S_{S}$ and receiver $R_{S}$ are assumed to have a circular sensing region with them being in the center. The secondary transmitter and receiver sensing regions are circular with each having radius $R_{r}$. The distance between them is $d$. They are further supposed to be communicating in the presence of primary users $A, B$ and $C$ as shown in Fig. 3. 


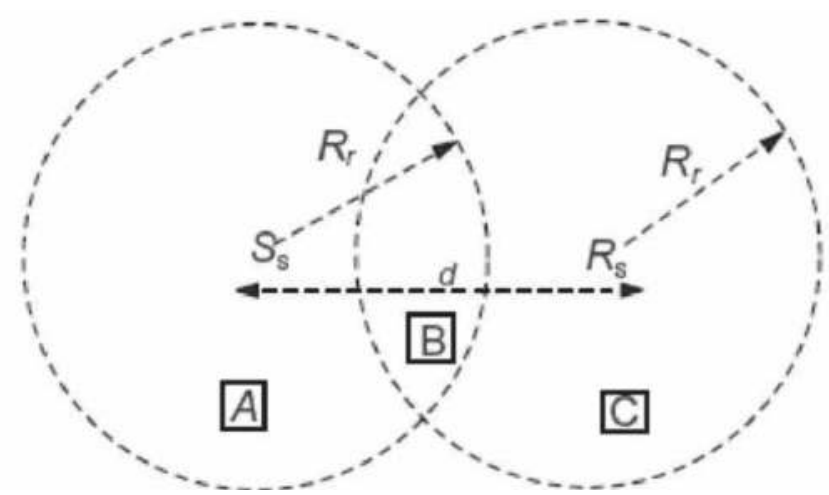

Fig. 3. The scenario for interference avoiding channel

The cognitive sender $S S$ can detect a spectral hole when both $A$ and $B$ are inactive whereas the secondary receiver $R S$ determines this when it finds both $B$ and $C$ to be not involved in a communication scenario. Since secondary transmitter and receiver do not have complete knowledge of primary users activity in each other's sensing regions, the spectral activity in their respective regions corresponds to the notion of being distributed. Similarly, the primary user activity sensed by the secondary transmitter-receiver pair continues to change with time i.e., different primary users become active and inactive at different time. Thus, the spectral activity is also assumed to be dynamic. To incorporate both these features, the conceptual model of Fig. 3 is reduced to the two switch mathematical model as shown in Fig. 4.

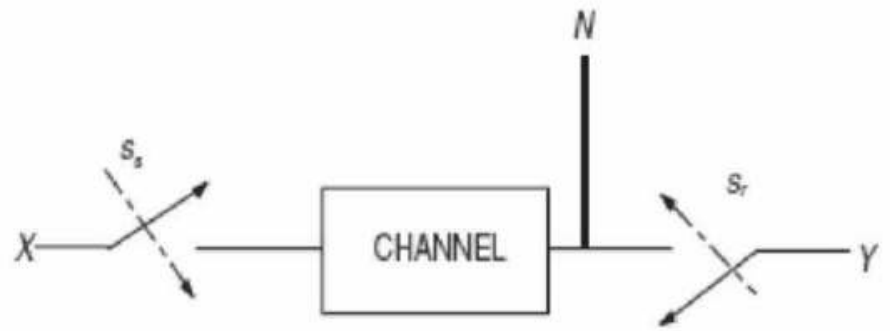

Fig. 4. The two switch model

The two switches $s_{s}, s_{r}$ are treated as binary random variables with $s_{s}, s_{r} \in\{0,1\}$. The value of $s_{s}=1$ or $s_{r}=1$ means that there are no primary users in the sensing region of the secondary transmitter or receiver and vice versa holds if either of these two values is zero. The input $X$ is related to the output 7 via following equation:

$$
Y=\left(X_{s_{S}}+N\right) s_{r}
$$

where $N$ is additive white Gaussian noise (AWGN) at the secondary receiver. $s_{r}$ is either 1 or 0 as mentioned above. So when it is multiplied as done in (7), it simply shows whether the secondary receiver has detected the primary device or not. 
If $s_{s}$ is only known to the transmitter and $s_{r}$ is only available to the receiver, the situation corresponds to communication with partial side information. Determination of capacity with partial side information involves input distribution maximization that is difficult to solve. This is not done and instead a tight capacity upper and lower bound is obtained for this communication channel. A capacity upperbound is determined by assuming that the receiver knows both $s s$ and $s r$ whereas, the transmitter only knows $\mathrm{s}_{\mathrm{s}}$. The expression of capacity $\mathrm{Cs}_{s}{ }^{*}$ of secondary user for this case is [23]:

$$
C_{s_{3, *}}(P)=\operatorname{Prob}\left[s_{s}=s_{T}=1\right] \log \left(1+\frac{P}{\operatorname{Prob}\left[s_{s}=1\right]}\right)
$$

where $P$ is the secondary transmitter power constraint. For the capacity lowerbound, [23] uses the results of [25]. For this a genie $g$ argument is used. It should be noted that utilization of genie concept represents the notion that either the sender or receiver is provided with some information noncausally. To determine the capacity lower bound the genie is supposed to provide some side information to the receiver.

So if the genie provides some information it must have an associated entropy rate. The results in [25] suggest that the improvement in capacity due to this genie information cannot exceed the entropy rate of the genie information itself. Using this argument and that the genie provides information to the receiver every $T$ channel uses, it is easy to establish that the capacity lower bound approaches the upper bound even for very highly dynamic environments.

It is assumed that the location of primary users in the system follows Poisson point process with a density of $X$ nodes per unit area. And that the primary user detection at the secondary transmitter and receiver is perfect. The capacity expression in (8), as given in [23], is evaluated to be:

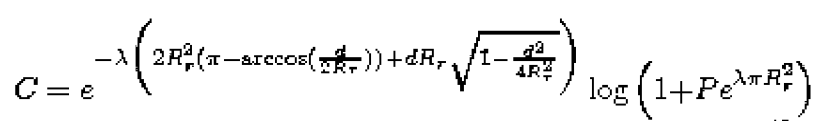

where, again, $P$ is the secondary power constraint.

\section{Colloborative Cognitive Channel}

In this channel, the cognitive user is modeled as a relay, with no information of its own, working between the primary transmitter and receiver. Capacity limits for collaborative communications have been recently explored [26] that suggest sufficient conditions on the geometry and the signal path loss of the transmitting entities for which performance close to the genie bound can be achieved [Natasha's tutorial].

Consider three nodes, source (s), relay $(\mathrm{r})$ and destination $(d)$ as illustrated in Fig. 5. 


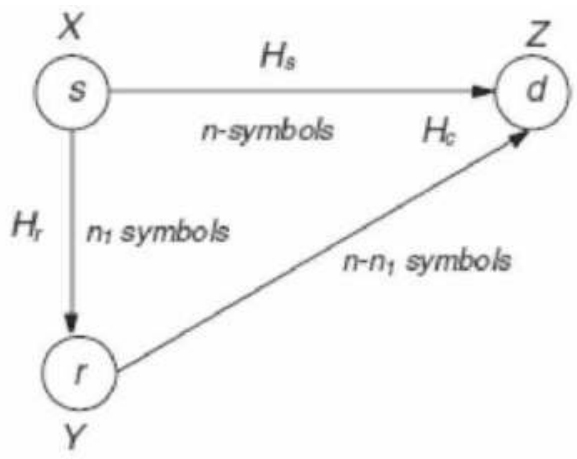

Fig. 5. The collaborative communication channel incorporating the source $s$, relay $r$ and the destination $d$ node

The relay node is assumed to work in half duplex mode, meaning that, it cannot receive and transmit data simultaneously. Thus the system works in two phases i.e., the listening phase and the collaborative phase. During the first phase the relay node receives data from the source node for the first $n 1$ transmissions while for the collaborative phase the relay transmits to the destination for the remaining $n-n_{1}$ transmissions, where $n$ are the number of channel uses, in which the source node wishes to transmit the $2^{n R}$ messages. Taking the channels as AWGN, considering $X$ and $U$ as column vectors representing the transmission from the source and relay node respectively and denoting by $Y$ and $Z$ the received messages at the relay and destination respectively, the listening phase is described via following equations:

$$
\begin{aligned}
& Z=H_{s} X+N_{z} \\
& Y=H_{r} X+N_{y}
\end{aligned}
$$

where $N_{z}$ and $N_{y}$ represent complex AWGN with variance $1 / 2, H_{s}$ is the fading matrix between the source and destination nodes and $H_{r}$ is the fading matrix between the source and relay nodes. In the collaborating phase:

$$
Z=H_{c}\left[X^{T}, U^{T}\right]^{T}+N_{z}
$$

where $H c$ is the channel matrix that contains $H s$ as a submatrix. It is well known that a Multiple Input Multiple Output (MIMO) system with Gaussian codebook and rate $R$ bits/channel can reliably communicate over any channel with transfer matrix $H$ such that $R \leq \log _{2} \operatorname{det}\left(I+H H^{\dagger}\right):=C(H)$ where $I$ denotes the identity matrix and $H^{\dagger}$ represents the conjugate transpose of $\mathrm{H}$. Before providing an explicit formula for rates, a little explanation is in order here.

During the first phase, relay listens for an amount of time $n 1$ and since it knows $H r$, it results in $n R \leq n_{1} C(H r)$. On the other hand, the destination node receives information at the rate of $\mathrm{C}(\mathrm{Hs})$ bits/channel during the first phase and at the rate of $\mathrm{C}\left(\mathrm{H}_{\mathrm{c}}\right)$ bits/channel during the second phase. Thus it may reliably decode the message provided that $n R \leq n_{1} C\left(H_{s}\right)+(n-$ 
$\left.\mathrm{n}_{1}\right) \mathrm{C}\left(\mathrm{H}_{\mathrm{c}}\right)$. Taking limit $n^{\wedge}><x>$ the ratio $\mathrm{n}_{1} / \mathrm{n}$ tends to a fraction $f$ such that the code of rate $R$ for the set of channels $\left(H_{r}, H_{c}\right)$ satisfies:

$$
\begin{gathered}
R \leq f C\left(H_{s}\right)+1(1-f) C\left(H_{c}\right) \\
R \leq f C\left(H_{r}\right)
\end{gathered}
$$

where $f \in[0,1]$

Similarly [23] presents a corollary which suggests that if the cognitive user has no message of its own, it can aid the primary system because it knows the primary's message, resulting in an improvement of primary user's data rates.

New outer bounds to the individual rates and the conditions under which these bounds become tight for the symmetric Gaussian cognitive radio (CR) channel in the low interference gain regime are presented in [27]. The CR transmitter is assumed to use dirty paper coding while deriving the outer bounds. The capacity of the CR channel in the low interference scenario is known when the CR employs "polite" approach by devoting some portion of its power to transmit primary user's (PU's) message. However, this approach does not guarantee any quality of service for the CR users. Hence, focus will be on the scenario when the CR goes for the "rude" approach, does not relay PU's message and tries to maximize its own rates only. It is shown that when both CR and the PU operate in low interference gain regime, then treating interference as additive noise at the PU receiver and doing dirty paper coding at the CR is nearly optimal.

\section{Comparsions}

The channel model presented in the first section uses complex coding techniques to mitigate channel interference that naturally results in higher throughput than that of the channel model of the second section. But there is one serious constraint in the first channel model, the information throughput of the cognitive user is highly dependent upon the distance between the primary transmitter and the cognitive transmitter. If this distance is large, secondary transmitter spends considerable time in obtaining the primary user's message. After obtaining and decoding the message, the cognitive device dirty paper codes it and sends it to its receiver. This message transfer from the primary to the cognitive transmitter results in lower number of bits transmitted per second by the cognitive radio and hence results in reduced data rates.

The capacity of the two switch model is independent of the distance between the two transmitters as the secondary transmitter refrains from sending data when it finds the primary user busy. Thus the benefit of the channel interference knowledge in the first channel model quickly disappears as the distance between the primary and secondary transmitter tends to increase. Accurate estimation of the primary system's message and the interference channel coefficient needed in the first channel model for dirty paper coding purposes is itself a problem. Inaccurate estimation of these parameters will result in a decrease in the rates of the cognitive radio because the dirty paper code, based on the knowledge of primary user's message and channel interference coefficient, will not be able to completely mitigate the primary user's interference. At the same time determination of 
channel interference coefficient requires a handshaking protocol to be devised which is a serious overhead and may result in poor performance.

On the other hand, the interference avoiding channel cannot overcome the hidden terminal problem. This problem naturally arises as the cognitive user would not be able to detect the presence of distant primary devices. The degraded signals from the primary users due to multipath fading and shadowing effects would further aggravate this problem.

This requires the secondary systems to be equipped with extremely sensitive detectors. But very sensitive detectors have prohibited long sensing times. Thus a protocol needs to be devised by virtue of which the sensed information is shared between the cognitive devices. Finally, the role of $C R$ as a relay in the third channel model restricts the real usefulness of the concept of dynamic spectrum utilization. Although limited, yet significant gains in data rates of the existing licensed user system can be obtained by restricting a CR device to relays of PU's message only.

\section{References}

1. Federal Communications Commission Spectrum Policy Task Force, "Report of the Spectrum Efficiency Working Group," Technical Report 02-135, no. November, 2002.

2. Shared Spectrum Company, "Comprehensive Spectrum occupancy measurements over six different locations," August 2005.

3. N. Devroye, P. Mitran, and V. Tarokh, "Achievable rates in cognitive radio channels," IEEE Transactions on Information Theory, vol. 52, no. 5, pp. 1813-1827, May 2006.

4. T. Han and K. Kobayashi, "A new achievable rate region for the interference channel," IEEE Transactions on Information Theory, vol. 27, no. 1, pp. 49-60, 1981.

5. S. I. Gel'fand and M. S. Pinsker, "Coding for channel with random parameters," Problems of Control and Information Theory, vol. 9, no. 1, pp. 19-31, 1980.

6. F. G. Awan and M. F. Hanif, "A Unified View of Information-Theoretic Aspects of Cognitive Radio," in Proc. International Conference on Information Technology: New Generations, pp. 327-331, April 2008.

7. S. Srinivasa and S. A. Jafar, "The throughput potential of cognitive radio - a theoretical perspective," IEEE Communications Magazine, vol. 45, no. 5, pp. 73-79, 2007.

8. Jovicic and P. Viswanath, "Cognitive radio: An information theoretic perspective," 2006 IEEE International Symposium on Information Theory, July 2006.

9. M. H. M. Costa, "Writing on dirty paper," IEEE Transactions on Information Theory, vol. 29, no. 3, pp. 439-441, May 1983.

10. Joseph Mitola, “Cognitive Radio: An Integrated Agent Architecture for Software Defined Radio," PhD Dissertation, KTH, Stockholm, Sweden, December 2000.

11. Paul J. Kolodzy, “Cognitive Radio Fundamentals," SDR Forum, Singapore, April 2005.

12. C.T.K.Ng and A. Goldsmith, "Capacity gain from transmitter and receiver cooperation," in Proc. IEEE Int. Symp. Inf. Theory, Sept. 2005.

13. N. Devroye, P. Mitran, and V. Tarokh, "Cognitive decomposition of wireless networks," in Proceedings of CROWNCOM, Mar. 2006.

14. Carleial, "Interference channels," IEEE Trans. Inf. Theory, vol. IT-24, no. 1, pp. 60-70, Jan. 1978.

15. N. Devroye, P. Mitran, and V. Tarokh, "Achievable rates in cognitive radio channels," IEEE Trans. Inf. Theory, vol. 52, no. 5, pp. 1813-1827, May 2006. 
16. W. Wu, S. Vishwanath, and A. Arapostathis, "On the capacity of the interference channel with degraded message sets," IEEE Trans. Inf. Theory, June 2006.

17. H. Weingarten, Y. Steinberg, and S. Shamai, "The capacity region of the Gaussian MIMO broadcast channel," IEEE Trans. Inf. Theory, vol. 52, no. 9, pp. 3936-3964, Sept. 2006.

18. N. Devroye, P. Mitran, and V. Tarokh, “Achievable rates in cognitive networks," in 2005 IEEE International Symposium on Information Theory, Sept. 2005.

19. E. C. van der Meulen, "Three-terminal communication channels," Adv. Appl. Prob., vol. 3, pp. 120-154, 1971.

20. I.Maric, R. Yates, and G. Kramer, "The strong interference channel with unidirectional cooperation," in Information Theory and Applications ITA Inaugural Workshop, Feb. 2006.

21. N. Devroye, P. Mitran, and V. Tarokh, "Achievable rates in cognitive radio channels," in 39th Annual Conf. on Information Sciences and Systems (CISS), Mar. 2005.

22. S. Jafar and S. Srinivasa, "Capacity limits of cognitive radio with distributed dynamic spectral activity," in Proc. of ICC, June 2006.

23. S. Srinivasa and S. A. Jafar, "The throughput potential of cognitive radio: A theoretical perspective," in Fortieth Asilomar Conference on Signals, Systems and Computers, 2006., Oct. 2006.

24. P. Cheng, G. Yu, Z. Zhang, H.-H. Chen, and P. Qiu, “On the achievable rate region of gaussian cognitive multiple access channel," IEEE Communications Letters, vol. 11, no.5, pp. 384-386, May. 2007.

25. S. A. Jafar, "Capacity with causal and non-causal side information-a unified view," IEEE Transactions on Information Theory, vol. 52, no. 12, pp. 5468-5474, Dec. 2006.

26. P. Mitran, H. Ochiai, and V. Tarokh, "Space-time diversity enhancements using collaborative communication," IEEE Transactions on Information Theory, vol. 51, no.6, pp. 2041-2057, June 2005.

27. F. G. Awan, N. M. Sheikh and F. H. Muhammad, "Outer Bounds for the Symmetric Gaussian Cognitive Radio Channel with DPC Encoded Cognitive Transmitter," in Proc. The World Congress on Engineering 2009 (WCE 2009) by the International Association of Engineers (IAENG), London, UK, July 2009. 


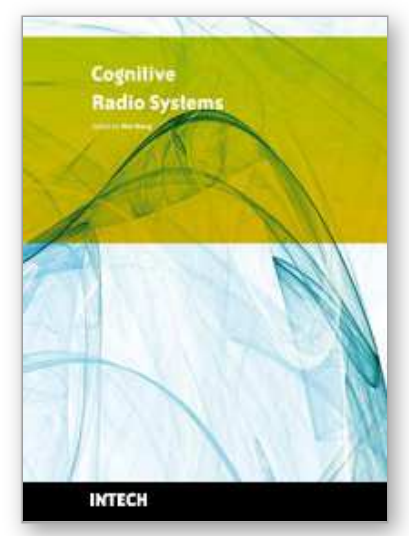

\section{Cognitive Radio Systems}

Edited by Wei Wang

ISBN 978-953-307-021-6

Hard cover, 340 pages

Publisher InTech

Published online 01, November, 2009

Published in print edition November, 2009

Cognitive radio is a hot research area for future wireless communications in the recent years. In order to increase the spectrum utilization, cognitive radio makes it possible for unlicensed users to access the spectrum unoccupied by licensed users. Cognitive radio let the equipments more intelligent to communicate with each other in a spectrum-aware manner and provide a new approach for the co-existence of multiple wireless systems. The goal of this book is to provide highlights of the current research topics in the field of cognitive radio systems. The book consists of 17 chapters, addressing various problems in cognitive radio systems.

\section{How to reference}

In order to correctly reference this scholarly work, feel free to copy and paste the following:

F. G. Awan, N. M. Sheikh and M. F. Hanif (2009). Information Theory of Cognitive Radio System, Cognitive Radio Systems, Wei Wang (Ed.), ISBN: 978-953-307-021-6, InTech, Available from:

http://www.intechopen.com/books/cognitive-radio-systems/information-theory-of-cognitive-radio-system

\section{INTECH}

open science | open minds

\section{InTech Europe}

University Campus STeP Ri Slavka Krautzeka 83/A

51000 Rijeka, Croatia

Phone: +385 (51) 770447

Fax: +385 (51) 686166

www.intechopen.com

\section{InTech China}

Unit 405, Office Block, Hotel Equatorial Shanghai

No.65, Yan An Road (West), Shanghai, 200040, China 中国上海市延安西路65号上海国际贵都大饭店办公楼 405 单元

Phone: +86-21-62489820

Fax: +86-21-62489821 
(C) 2009 The Author(s). Licensee IntechOpen. This chapter is distributed under the terms of the Creative Commons Attribution-NonCommercial-ShareAlike-3.0 License, which permits use, distribution and reproduction for non-commercial purposes, provided the original is properly cited and derivative works building on this content are distributed under the same license. 